\title{
Macrophages are required for neonatal heart regeneration
}

\author{
Arin B. Aurora, ${ }^{1}$ Enzo R. Porrello, ${ }^{1}$ Wei Tan, ${ }^{1}$ Ahmed I. Mahmoud, ${ }^{2}$ Joseph A. Hill, ${ }^{2}$ \\ Rhonda Bassel-Duby, ${ }^{1}$ Hesham A. Sadek, ${ }^{2}$ and Eric N. Olson ${ }^{1}$
}

1Department of Molecular Biology and 2Department of Internal Medicine, University of Texas Southwestern Medical Center, Dallas, Texas, USA.

\begin{abstract}
Myocardial infarction (MI) leads to cardiomyocyte death, which triggers an immune response that clears debris and restores tissue integrity. In the adult heart, the immune system facilitates scar formation, which repairs the damaged myocardium but compromises cardiac function. In neonatal mice, the heart can regenerate fully without scarring following MI; however, this regenerative capacity is lost by P7. The signals that govern neonatal heart regeneration are unknown. By comparing the immune response to MI in mice at P1 and P14, we identified differences in the magnitude and kinetics of monocyte and macrophage responses to injury. Using a cell-depletion model, we determined that heart regeneration and neoangiogenesis following MI depends on neonatal macrophages. Neonates depleted of macrophages were unable to regenerate myocardia and formed fibrotic scars, resulting in reduced cardiac function and angiogenesis. Immunophenotyping and gene expression profiling of cardiac macrophages from regenerating and nonregenerating hearts indicated that regenerative macrophages have a unique polarization phenotype and secrete numerous soluble factors that may facilitate the formation of new myocardium. Our findings suggest that macrophages provide necessary signals to drive angiogenesis and regeneration of the neonatal mouse heart. Modulating inflammation may provide a key therapeutic strategy to support heart regeneration.
\end{abstract}

\section{Introduction}

The adult mammalian heart possesses limited potential for repair and regeneration. Ischemic injury during myocardial infarction (MI) causes death of cardiomyocytes, followed by an inflammatory response and replacement of lost myocardium with a fibrotic scar. As a consequence, $\mathrm{MI}$ often leads to heart failure, the cause of 1 in every 4 deaths in the United States (1). Since efficient regeneration has not been demonstrated in adult mammalian myocardium, immense effort has been focused on therapies to enhance infarct healing.

We recently demonstrated that the neonatal mouse heart can regenerate fully following apical resection (2) and MI (3), but this regenerative potential is lost by P7. Zebrafish, in contrast, can regenerate heart tissue throughout life, in part because cardiomyocytes in this organism are mononucleated and remain proliferative into adulthood (4). Mammals are born with proliferative, mononucleated cardiomyocytes, which undergo a final round of replication without cell division, leaving the majority of cardiomyocytes binucleated $(5,6)$. The proliferative capacity of neonatal cardiomyocytes likely contributes to the regenerative capacity of the newborn mouse heart. However, the signals that trigger the regenerative process are largely unknown.

Neonatal heart regeneration is accompanied by robust infiltration of inflammatory cells as well as upregulation of many genes linked to inflammation (2). Interestingly, the immune system continues to mature after birth, and many of the changes in cytokine expression and cellular functions that occur coincide with the period of neonatal cardiac regeneration. In mice, the adaptive immune response does not begin to mature until 3 weeks of age (7-13), and the innate immune response is quantitatively and qualitatively different in the early neonate and adult (14-17). However, the immune response to injury is not well characterized during this period of postnatal development.

Conflict of interest: The authors have declared that no conflict of interest exists. Citation for this article: J Clin Invest. 2014;124(3):1382-1392. doi:10.1172/JCI72181.
Inflammation can impede recovery of the adult heart from injury (18). Recent studies demonstrated that disruption of C/ EBP signaling in the adult epicardium reduced injury-induced neutrophil infiltration and improved cardiac function (19). However, both positive (20-22) and negative (23-26) correlations between tissue remodeling and monocytes, and their lineage descendant macrophages, have been reported. CCL2, a chemokine that recruits and activates monocytes, seems to play a dual role in MI. Targeted deletion of the CCL2 receptor (CCR2) improved left ventricular dilation and dysfunction $(20,21)$, while cardiac overexpression of CCL2 also improved outcome (23) by inducing macrophages infiltration, angiogenesis, myocardial IL-6 secretion, and accumulation of cardiac myofibroblasts. Biphasic recruitment of $\mathrm{Ly}-6 \mathrm{Chi}$ and $\mathrm{Ly}-6 \mathrm{C}^{\mathrm{lo}}$ monocyte subsets from the spleen also promotes infarct healing (26-29).

Multiple observations indicate an inverse relationship between the development of the immune system and epimorphic regeneration in lower vertebrates and support the longstanding hypothesis that the mammalian immune system constrains regenerative capacity (30). Recently, macrophage infiltration was demonstrated to be essential for limb regeneration in newts (31), suggesting that this inflammatory process represents an evolutionarily conserved mechanism for tissue repair. Interestingly, the developmental timing of immune system maturation and terminal differentiation of cardiomyocytes are coupled, suggesting that immunity might influence cardiac regenerative capacity in mammals. We sought to understand the nature of the neonatal immune response to injury in the context of cardiac regeneration. Comparison of the immune response to MI in P1 (regenerative) and P14 (nonregenerative) mice revealed differences in several cell types, including monocytes/macrophages. We focused further on monocytes/macrophages, given that they are central to outcome after MI in adults and can promote mammalian regeneration of skeletal muscle and the nervous system $(32,33)$. By utilizing a model of mono- 
A
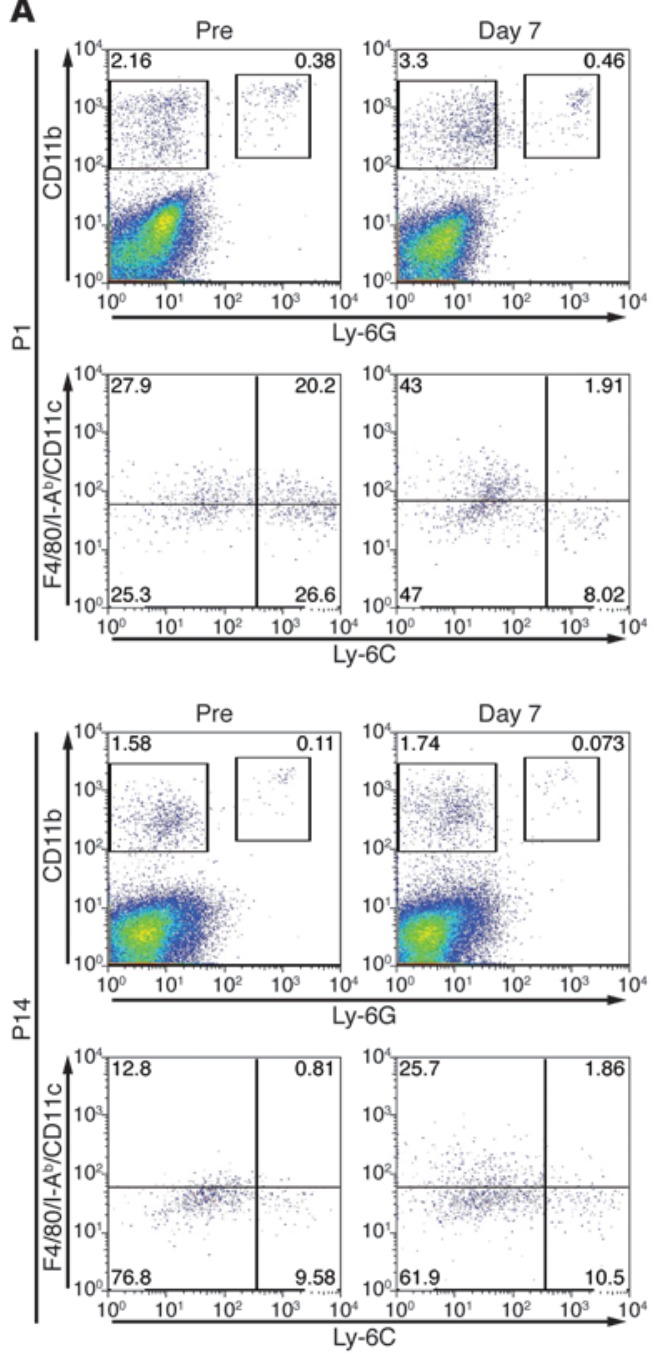

B Mononuclear phagocytes

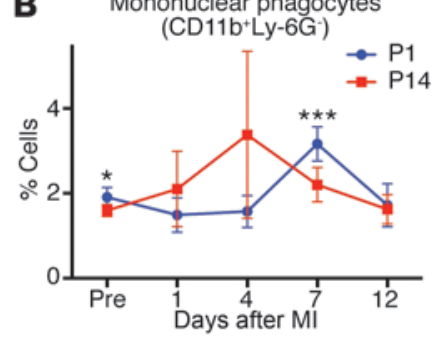

$M \Phi / D C$

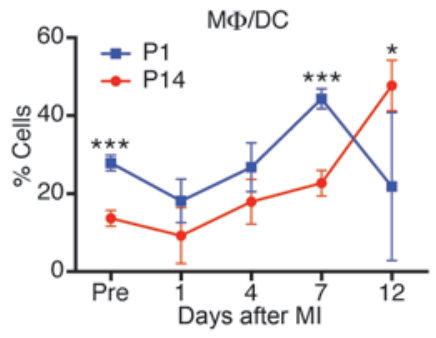

C

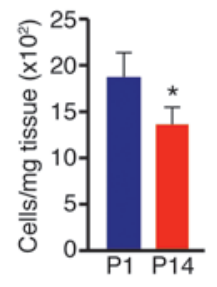

D
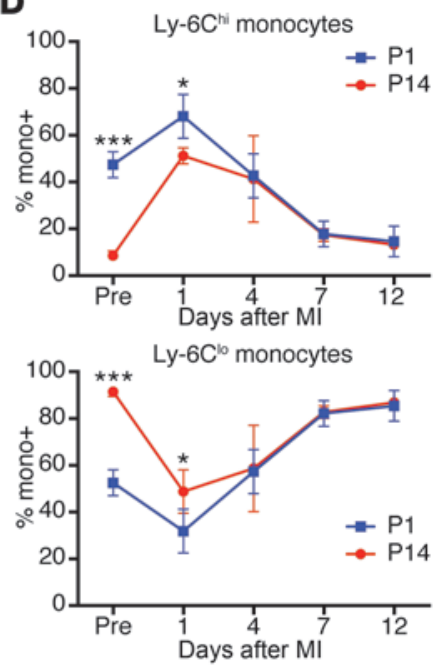
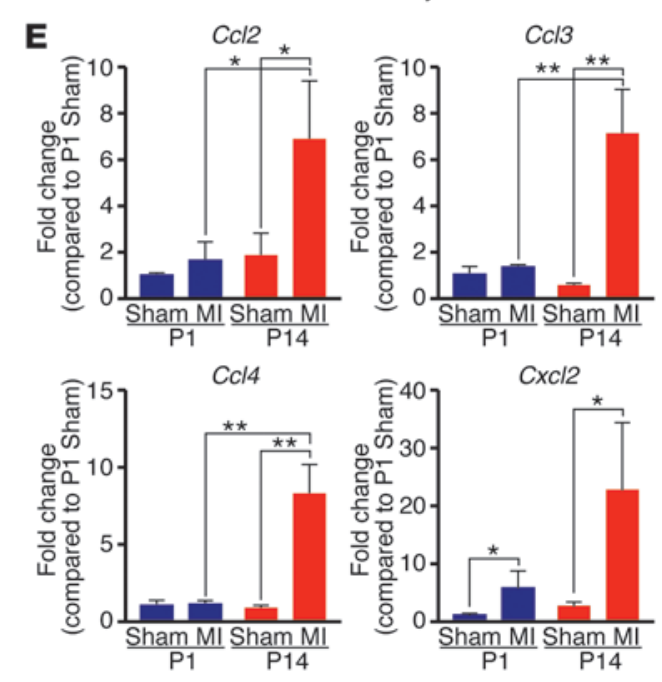

\section{Figure 1}

Mononuclear phagocytes respond differently to MI in P1 and P14 mice. (A) Single cell suspensions isolated from hearts of mice before MI (Pre) or 7 days following MI at P1 or P14 were stained with anti-CD11b, -Ly-6G, -Ly-6C, -F4/80, -CD11c, -I-Ab mAbs and analyzed by FACS. Mononuclear phagocytes were identified as CD11b+Ly-6G- and neutrophils were identified as CD11b+Ly-6G+ (top panels). Within the mononuclear phagocyte population, macrophages/DCs are classified as (F4/80/CD11 c/l-A $\left.)^{\mathrm{b}}\right)^{\text {hi }} \mathrm{Ly}-6 \mathrm{C}^{\mathrm{lo}}$ and monocytes are depicted as $\left(\mathrm{F} 4 / 80 / C D 11 \mathrm{C} / \mathrm{l}-\mathrm{A}^{\mathrm{b}}\right)^{\mathrm{lo}} \mathrm{Ly}-6 \mathrm{C}^{\mathrm{hi}}$ or $(\mathrm{F} 4 / 80 /$ $\left.\mathrm{CD} 11 \mathrm{c} / \mathrm{l}-\mathrm{A}^{\mathrm{b}}\right)^{\mathrm{lo}} \mathrm{Ly}-6 \mathrm{C}^{\mathrm{lo}}$ (bottom panels). Percentages of cells are indicated for the representative dot plots. (B) Quantification at the indicated time points to compare the percentage of all mononuclear phagocytes or macrophages/DCs (M $\Phi / D C s)$ relative to the leukocyte-enriched gate or mononuclear phagocyte population, respectively, in mice undergoing MI at P1 (blue) or P14 (red) ( $n=3-5$ per time point). (C) Total number of mononuclear phagocytes per milligram of heart tissue 7 days after MI at P1 or P14. (D) Relative percentages of Ly-6Chi and Ly-6Clo monocytes in the heart were quantified over time following Ml of mice at P1 or P14 ( $n=3-5$ per time point). (E) Real-time RT-PCR analysis of cardiac chemokine expression at 3 days following Ml of P1 or P14 mice. Expression is relative to that in P1 sham-operated mice [P1 Sham]) $(n=3)$. Data are mean \pm SEM. ${ }^{*} P<0.05,{ }^{*} P<0.01,{ }^{* *} P<0.001$.

cyte/macrophage depletion in P1 mice, we show that monocytes/ macrophages are required for neonatal heart regeneration and efficient neoangiogenesis. Our findings suggest that therapeutic modulation of monocytes/macrophages will facilitate regeneration of the mammalian heart.

\section{Results}

The immune response to MI differs between $P 1$ and P14 mice. To understand differences between the immune response during and beyond the regenerative period, we profiled the cellular response to $\mathrm{MI}$ in P1 and P14 mice over time by FACS. MI was induced in mice by permanent ligation of the left anterior descending coronary artery. Before MI or at 1, 4, 7, or 12 days after MI, pooled hearts or spleens were enzymatically digested or mechanically disrupted, respectively, to single cell suspensions and stained with antibodies to distinguish neutrophils (CD11 b $\left.\mathrm{by}^{+} 6 \mathrm{G}^{+}\right)$; mononuclear phagocytes (CD11 $\left.\mathrm{b}^{+} \mathrm{Ly}^{-} 6 \mathrm{G}^{-}\right)$; $\mathrm{T}$ lymphocytes (TCR $\left.\beta^{+} \mathrm{B} 220^{-}\right)$; and B lymphocytes (TCR $\beta^{-}$B $220^{+}$) (Figure 1 , A and B, and Supplemental Figures 1-3; supplemental material available online with this article; doi:10.1172/JCI72181DS1).

Representative FACS plots illustrate gating to define neutrophils and mononuclear phagocytes in heart samples before MI or 7 days 
A
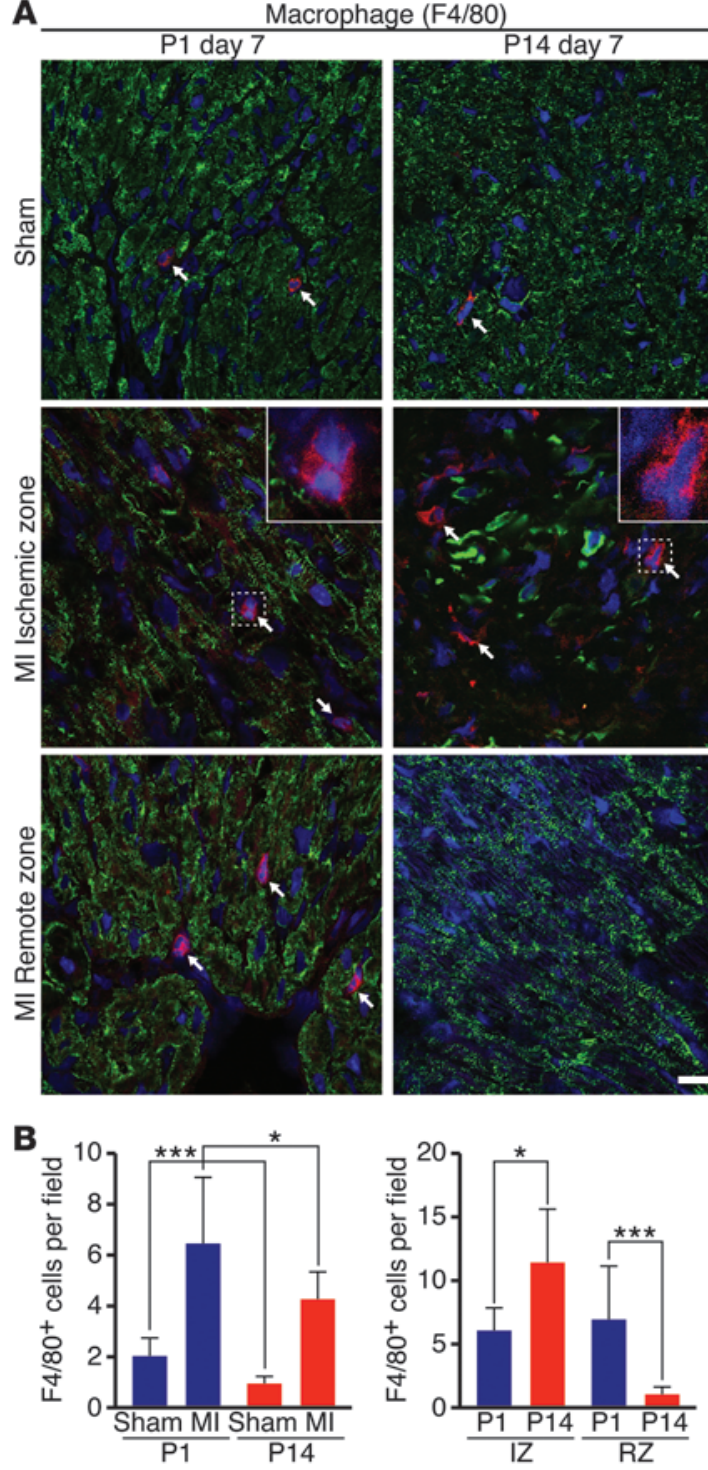

following MI at P1 or P14 (Figure 1A), and dynamic alterations over time were quantified as a percentage of the leukocyte-enriched live gate, determined by characteristic forward/side scatters (Figure 1B and Supplemental Figure 1A). Additional staining of the mononuclear phagocyte population allowed us to further delineate Ly6C $^{\mathrm{lo}}\left(\mathrm{F} 4 / 80 / \mathrm{I}-\mathrm{A}^{\mathrm{b}} / \mathrm{CD} 11 \mathrm{c}\right)^{\mathrm{hi}}$ macrophages/DCs, Ly6C ${ }^{\mathrm{lo}}\left(\mathrm{F} 4 / 80 / \mathrm{I}-\mathrm{A}^{\mathrm{b}} /\right.$ CD11c $)^{\text {lo }}$ monocytes, and Ly6C ${ }^{\text {hi }}\left(\mathrm{F} 4 / 80 / \mathrm{I}-\mathrm{A}^{\mathrm{b}} / \mathrm{CD} 11 \mathrm{c}\right)^{\text {lo }}$ monocytes (Figure 1A) according to established methods (28).

Mononuclear phagocyte abundance was relatively increased in the heart in the absence of injury and at 7 days following MI at P1 compared with P14 (Figure 1B). Absolute numbers of mononuclear phagocytes in the myocardium at 7 days following MI confirmed there was an increase of mononuclear phagocytes in the P1 group (Figure 1C). Furthermore, the quantity of macrophages/DCs was higher prior to MI and at 7 days after MI (Figure 1B), a period during which cardiomyocyte proliferation and the regenerative process are robustly active (3). We also quantified the relative percentage of Ly- $6 \mathrm{C}^{\text {hi }}$ or Ly- $6 \mathrm{C}^{\text {lo }}$ monocytes infiltrating the heart. While P1 hearts showed an increased percentage of

\section{Figure 2}

Macrophage abundance and localization in hearts following $\mathrm{Ml}$ of $\mathrm{P} 1$ or P14 mice. (A) Heart sections were stained with anti-F4/80 (red) to visualize macrophages in sham-operated (Sham) P1 or P14 mice or in the IZ or RZ of hearts 7 days following MI. Myocardium is labeled with desmin (green), and nuclei are visualized with Hoechst (blue). Arrows point to $\mathrm{F} 4 / 80^{+}$macrophages. Insets shows high-magnification images of $\mathrm{F} 4 / 80^{+}$cells. Scale bar: $20 \mu \mathrm{m}$. (B) The total number of $\mathrm{F} 4 / 80^{+}$macrophages 7 days following $\mathrm{Ml}$ or sham surgery was quantified on 3 serial sections per heart starting at suture level (or plane of suture for shamoperated mice) and progressing toward the apex $(n=3-4$ mice per group). The P1 group had more total macrophages than the P14 group in the absence of injury (Sham) and after MI. The number of F4/80+ macrophages was also compared within the IZ or RZ to reveal localization differences. Data represent mean \pm SEM. ${ }^{*} P<0.05,{ }^{* * *} P<0.001$.

Ly-6Chi monocytes compared with P14 hearts before and at 1 day following MI, both groups had a biphasic, sequential infiltration of Ly- $6 \mathrm{C}^{\text {hi }}$ and Ly- $6 \mathrm{C}^{\text {lo }}$ monocytes into the heart in response to infarction (Figure 1D), as previously described for the adult mouse (28). Comparisons were also made in the spleen, a major source of monocyte reserves following myocardial injury. While baseline percentages of all mononuclear phagocytes and Ly-6Chi monocytes were higher in P1 mice, the abundance was similar between groups at multiple time points following MI at P1 or P14 (Supplemental Figure 2, A and B). Given the differences in the magnitude of mononuclear phagocytes, we compared the expression level of monocytic chemokines in the heart 3 days following sham surgery or MI at P1 or P14 to examine possible differences in recruitment signals. Interestingly, the robust upregulation of expression that occurs following MI at P14 was not present for most chemokines following MI at P1, suggesting that these signals are not the mechanism for cell recruitment or activation (Figure 1E).

Neutrophil numbers peaked at day 1 following MI at P1 and P14 (Supplemental Figure 1A), consistent with established knowledge of neutrophil responses. While neutrophil abundance was relatively higher in the heart at many time points following $\mathrm{MI}$ at P1 compared with that at P14 (Supplemental Figure 1A), we did not initially focus on this cell type due to its transient presence at the injury site.

Given that the lymphocyte response is relatively weak and underdeveloped at birth, FACS was not a reproducible method to quantify the low abundance of $\mathrm{T}$ and $\mathrm{B}$ lymphocytes in the heart following MI at P1. Therefore, we compared lymphocyte responses to $\mathrm{MI}$ at P1 and P14 by quantifying them in the spleen, a secondary lymphoid organ and major site of lymphocyte accumulation and activation. Representative FACS plots from spleens before injury or at 7 days following MI at P1 or P14 illustrate the gating used to differentiate B cells, T cells, and T cell subsets (Supplemental Figure 3, A and B). B cells and T cells were generally present at higher percentages in P14 spleens before and after injury (Supplemental Figure 3C) compared with P1 spleens. Furthermore, within the $\mathrm{TCR}^{+}$population of the spleen, $\mathrm{P} 1$ mice had a significantly lower percentage of $\mathrm{CD}^{+} \mathrm{T}$ cells and $\mathrm{CD} 25^{+} \mathrm{CD} 4^{+}$regulatory $\mathrm{T}$ cells (Supplemental Figure 3D), consistent with the established observation that regulatory T cells do not emerge until after P4 (13). Instead, the majority of $\mathrm{T}$ cells from the $\mathrm{P} 1$ group were $\mathrm{CD} 25^{+} \mathrm{CD} 4^{-}$and likely represent an immature state (Supplemental Figure 3D).

Localization of inflammatory cells. To confirm differences in the P1 and P14 monocyte/macrophage response, we sought to enumerate immune cell types in heart sections using immunostaining. To 
A

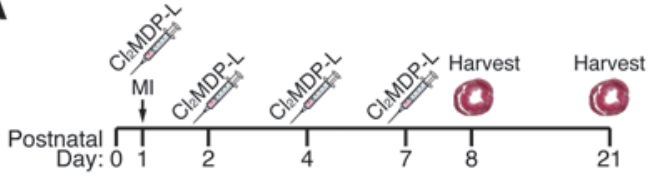

B

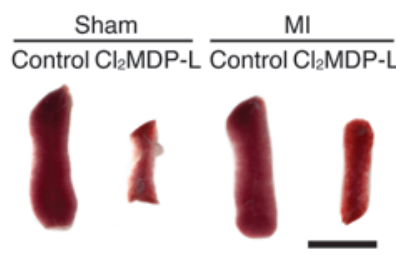

C

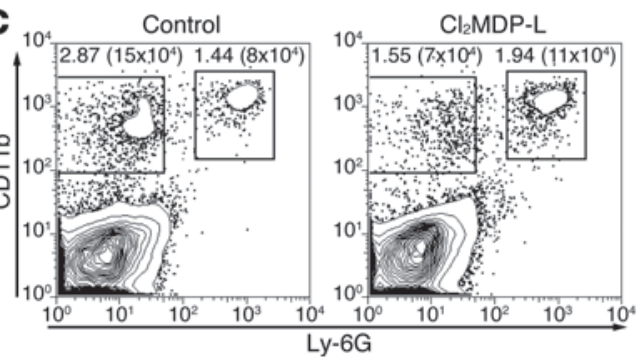

D

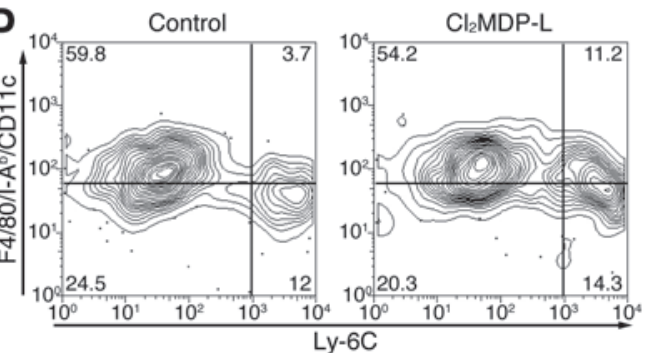

E

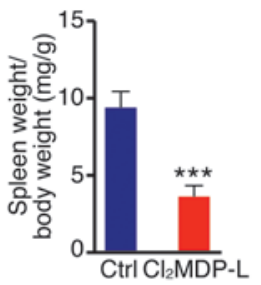

F
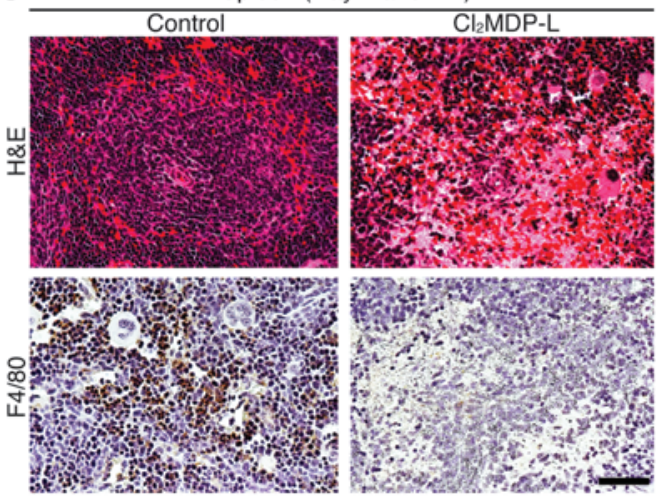

G

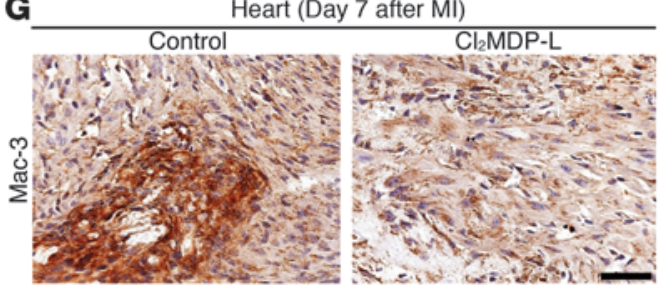

\section{Figure 3}

Depletion of monocytes/macrophages in a model of neonatal heart regeneration. (A) Experimental strategy to deplete monocytes/macrophages using clodronate liposomes ( $\mathrm{Cl}_{2} \mathrm{MDP}-\mathrm{L}$ ) following MI at P1. (B) Spleens harvested from control or monocyte/macrophage-depleted mice 3 days following sham or MI surgeries are shown. Scale bar: $4 \mathrm{~mm}$. (C) Contour plots from mice 3 days after MI depict mononuclear phagocyte and neutrophil percentages and numbers in hearts after depleting with $\mathrm{Cl}_{2} \mathrm{MDP}$ - $\mathrm{L}$ or injection of saline (control). (D) Mononuclear phagocytes were further specified as Ly- $6 \mathrm{C}^{\text {hi }}$ monocytes (bottom right), Ly- $6 \mathrm{C}^{l o}$ monocytes (bottom left), and macrophages/DCs (top left). (E) By 7 days after MI, spleens weigh significantly less in $\mathrm{Cl}_{2} \mathrm{MDP}-\mathrm{L}-$ treated neonates compared with those in control mice. Data represent mean $\pm \mathrm{SEM}$. ${ }^{* * *} P<0.0001$. $(\mathbf{F})$ $\mathrm{H} \& \mathrm{E}$-stained sections of the spleen and immunohistochemistry with $\mathrm{F} 4 / 80$ show that $\mathrm{Cl}_{2} \mathrm{MDP}$-L-treated mice have been depleted of monocytes/ macrophages 7 days following MI. Scale bar: $40 \mu \mathrm{m}$. (G) Immunohistochemical staining for Mac-3 depicts monocyte/macrophage depletion in the $\mathrm{IZ}$ of heart sections from $\mathrm{Cl}_{2} \mathrm{MDP}$-L-treated mice compared to control mice. Scale bar: $40 \mu \mathrm{m}$.

assess possible heterogeneity in cellular localization, we analyzed regions both in the ischemic zone (IZ) and the remote zone (RZ) of transverse heart sections (Figure 2A). Serial heart sections, beginning at the ligature and progressing through the apex, were stained for markers specific for macrophages (F4/80) (Figure 2A) and neutrophils (NIMP-R14) (Supplemental Figure 1B). Sections from the area of the heart in which left anterior descending ligation was performed were also taken from sham-operated mice and stained for F4/80. Representative images (Figure $2 \mathrm{~A}$ ) and quantification of the total number of $\mathrm{F} 4 / 80^{+}$macrophages (Figure $2 \mathrm{~B}$ ) showed significantly more cells in hearts at P1 compared with P14 in the absence of ischemic injury. Seven days following MI at P1, macrophages appeared more abundant in the heart overall and were uniformly distributed throughout the myocardium (Figure 2A). Quantification of the number of macrophages localized to the IZ or RZ confirmed this observation (Figure 2B). In contrast, hearts from mice 7 days following MI at P14 had fewer macrophages overall that were almost exclusively localized to the IZ (Figure 2, A and B). In both the $\mathrm{P} 1$ and $\mathrm{P} 14$ groups, neutrophils were present in small numbers in both the IZs and RZs of the myocardia (Supplemental Figure 1, $\mathrm{B}$ and $\mathrm{C}$ ). Together with the FACS data, the immunostaining shows that, in regenerating hearts ( $\mathrm{P} 1$ group), there is an increased abundance of macrophages and a striking difference in macrophage localization compared with nonregenerating hearts (P14 group). Therefore, macrophages may function differently in the regenerating neonatal myocardium following MI.

Depleting macrophages in the neonatal MI model. Significant regeneration takes place by 7 days following MI at P1 $(3,34)$. Within the first week following MI at P1, monocytes/macrophages had the highest, consistent representation in the heart of the cell types assessed and have been shown to have regenerative effects in other systems $(32,33)$. We hypothesized that, because of their distinc- 
A

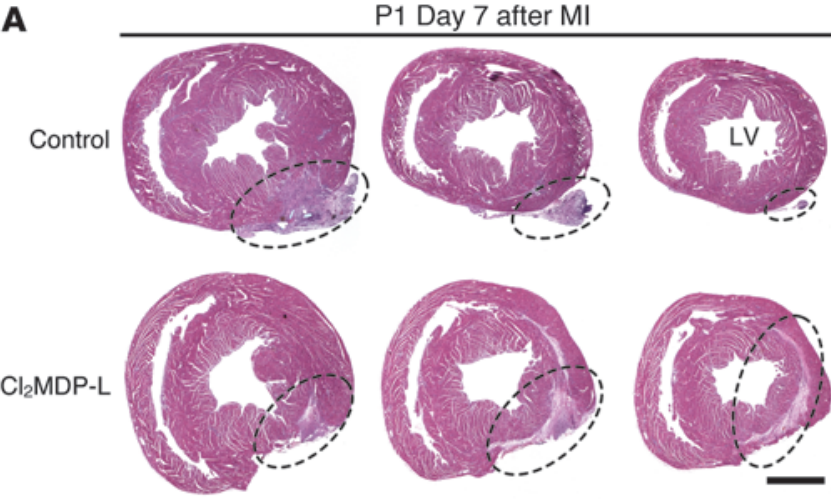

B

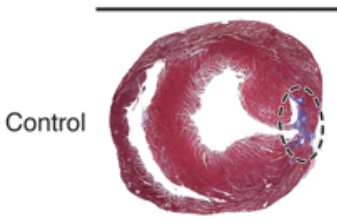

P1 Day 21 after MI

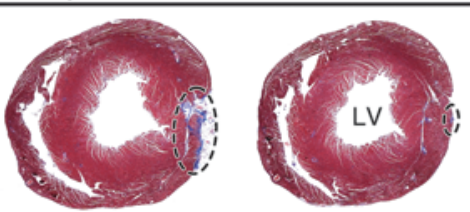

$\mathrm{Cl}_{2} \mathrm{MDP}-\mathrm{L}$
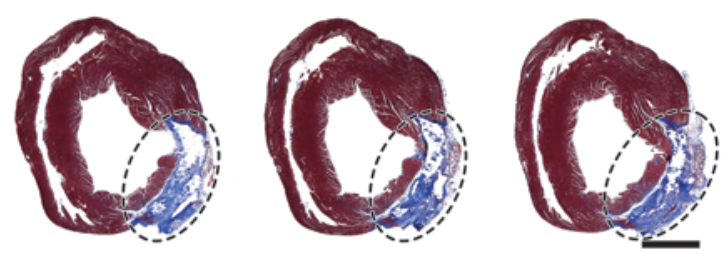

C

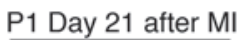

Control

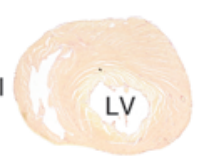

$\mathrm{Cl}_{2} \mathrm{MDP}-\mathrm{L}$

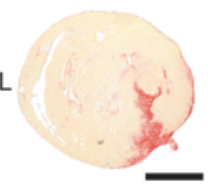

D

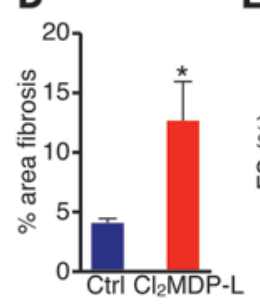

E

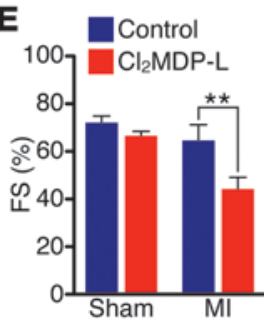

tive abundance and localization, monocytes/macrophages from mice undergoing MI at P1 may have a role in heart regeneration that is different from the role of monocytes/macrophages in nonregenerative MI. To test the role of monocytes/macrophages, we applied a method for clodronate liposome-mediated depletion of monocytes/macrophages to the neonatal MI model (24). Following i.v. injection of liposomes containing clodronate, phagocytosis by monocytes/macrophages results in apoptosis and subsequent depletion of the monocyte/macrophage population. We adapted this method to neonatal MI (Figure 3A). By day 3 after MI, spleens from clodronate liposome-treated $\left(\mathrm{CL}_{2} \mathrm{MDP}-\mathrm{L}\right.$-treated) mice were already visibly smaller than those of control mice (Figure 3B), and FACS analysis of hearts showed a specific depletion of the mononuclear phagocyte $\left(\mathrm{CD} 11 \mathrm{~b}^{+} \mathrm{Ly}-6 \mathrm{G}^{-}\right)$population (Figure 3C). Compared with control-treated neonates, $\mathrm{P} 1$ mice given $\mathrm{CL}_{2} \mathrm{MDP}-\mathrm{L}$ had more than a $50 \%$ reduction in cardiac mononuclear phagocytes but no depletion of neutrophils (Figure 3C). Additional staining of the remaining mononuclear phagocyte population

\section{Figure 4}

Depletion of monocytes/macrophages blocks heart regeneration in neonatal mice. (A) At 7 days after MI, H\&E-stained serial heart sections, starting below the ligature and progressing toward the apex ( 3 sections per heart), show a diminishing infarct area that has been displaced to the periphery in control mice. $\mathrm{Cl}_{2} \mathrm{MDP}-\mathrm{L}-$ treated mice maintain an interstitial scar. The injured area is circled. Scale bar: $1 \mathrm{~mm}$. (B) Masson's trichrome staining of serial sections, starting below the ligature and progressing toward the apex, from control or $\mathrm{Cl}_{2} \mathrm{MDP}-\mathrm{L}-$ treated mice 21 days following $\mathrm{Ml}$ at $\mathrm{P} 1$ to visualize fibrosis. The injured area is circled. Scale bar: $1 \mathrm{~mm}$. (C and $\mathbf{D})$ Picrosirius red staining on heart sections from control or $\mathrm{Cl}_{2} \mathrm{MDP}$-L-treated mice 21 days following MI at P1. (C) Representative images and (D) quantification as a percentage of total section area ( $\geq 7$ sections per heart) show significantly more fibrosis in the $\mathrm{Cl}_{2} \mathrm{MDP}$-L-treated group $(n=7-8$ mice per group). Scale bar: $1 \mathrm{~mm}$. (E) Cardiac function following $\mathrm{Ml}$ or sham surgeries was assessed at 28 days after MI by echocardiography. Data are expressed as the percentage of FS ( $n=3-4$ mice per group). Data are mean \pm SEM. ${ }^{\star} P<0.05,{ }^{\star \star} P<0.01$.

showed that the relative ratio of macrophages/DCs and Ly- $6 \mathrm{C}^{\mathrm{hi}}$ or Ly-6C $\mathrm{C}^{\text {lo }}$ monocytes was unchanged (Figure 3D). By day 7 after $\mathrm{MI}, \mathrm{CL}_{2} \mathrm{MDP}-\mathrm{L}-$ treated spleens were significantly smaller, as a ratio to body weight (Figure 3E), and lacked the morphologically distinct red pulp and white pulp compartments that were visible in controls by $\mathrm{H} \& \mathrm{E}$ staining (Figure $3 \mathrm{~F}$ ). Immunohistochemical detection of the macrophage markers F4/80 in the spleen (Figure 3F) and Mac-3 in heart (Figure 3G) showed a marked reduction in the macrophage population.

Regeneration depends on macrophages. Next, we determined the ability of P1 mice depleted of monocytes/macrophages to regenerate their hearts in response to MI. By day 7 after MI, control mice already exhibited substantial regeneration (Figure 4A). H\&E staining of sections at and below the ligature revealed replacement of dead myocardia and subsequent marginalization of the remaining scar to the tissue periphery in control mice (Figure 4A, left to right). In contrast, sections from mice treated with $\mathrm{CL}_{2} \mathrm{MDP}-\mathrm{L}$ had visible transmural IZs that extended toward the apex at day 7 after MI, suggesting that regeneration was interrupted within the first week following MI (Figure 4A).

By 21 days following MI at P1, control mice had completely regenerated their myocardia from the ligature to the apex. Masson's trichrome and Picrosirius red staining for collagens showed that only a small, localized fibrotic response around the ligation site was visible in some control samples (Figure 4, B and C). Strikingly, the $\mathrm{CL}_{2} \mathrm{MDP}-\mathrm{L}-$ treated group showed visible collagen staining throughout the infarct area to the apex. Furthermore, the area of fibrotic tissue quantified by Picrosirius red staining was significantly higher in $\mathrm{CL}_{2} \mathrm{MDP}-\mathrm{L}$-treated mice, suggesting that regeneration was monocyte/macrophage dependent (Figure 4D). Consistent with increased fibrotic scar formation, cardiac function was significantly depressed in $\mathrm{CL}_{2} \mathrm{MDP}-\mathrm{L}$-treated mice following MI compared with that in saline controls or sham-operated mice treated with $\mathrm{CL}_{2} \mathrm{MDP}-\mathrm{L}$, further supporting a role for monocytes/ macrophages in functional regeneration of the neonatal heart after MI (Figure 4E).

Monocyte/macrophage populations differ between P1 and P14 mice. It has been well documented that adult macrophages mediate scar formation $(24,35,36)$, not regeneration, following ischemic heart injury. Therefore, we reasoned that monocytes/macrophages from P1 mice must differ functionally from P14 monocytes/macro- 
A

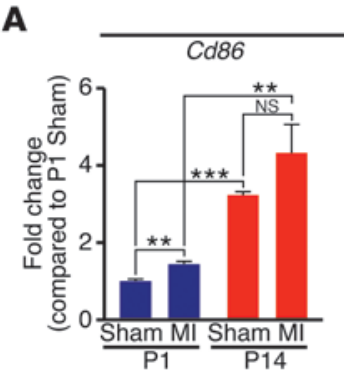

M1 macrophage
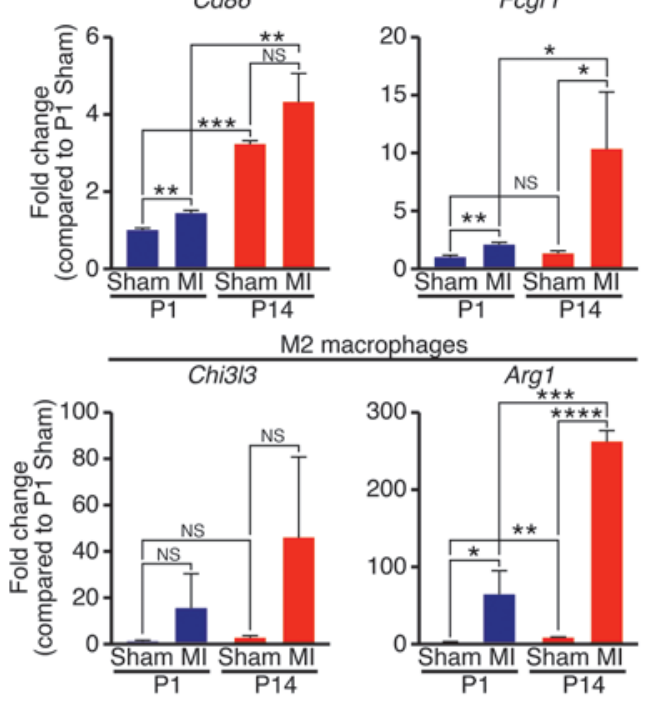

B

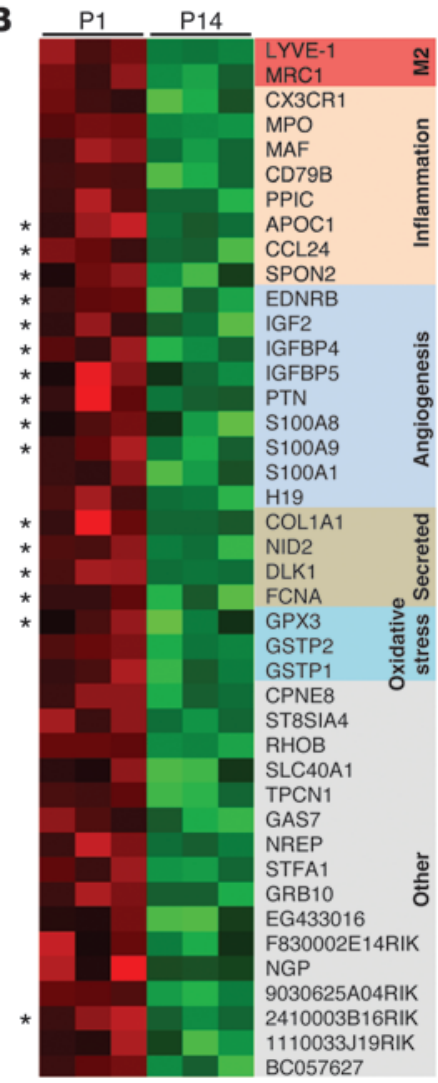

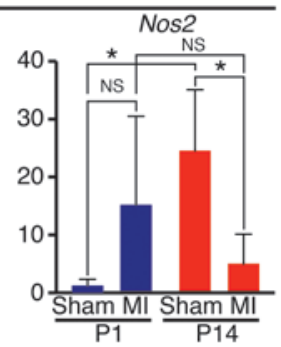

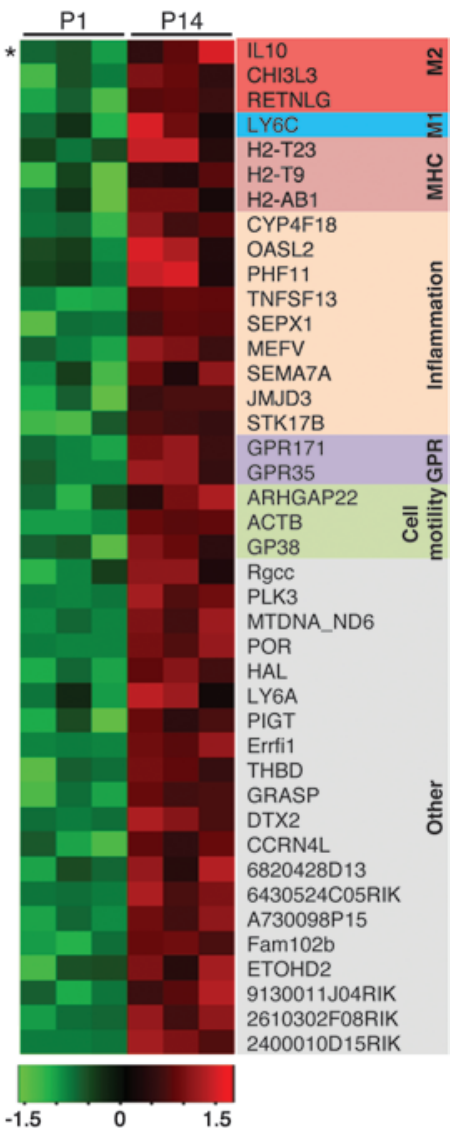

\section{Figure 5}

Macrophage immunophenotyping in $\mathrm{P} 1$ and $\mathrm{P} 14$ mice. (A) Real-time qPCR analysis of M1 and M2 macrophage genes in hearts from mice 3 days after sham surgery or MI at P1 or P14 $(n=3)$. Data represent mean \pm SEM. ${ }^{*} P<0.05,{ }^{*} P<0.01$, ${ }^{* * *} P<0.001,{ }^{* * * *} P<0.0001$. (B) Expression profiling during the macrophage response to $\mathrm{Ml}$ in $\mathrm{P} 1$ or $\mathrm{P} 14$ mice identified multiple genes that are differentially expressed in purified cardiac macrophages. The heat map shows mRNAs changed 2-fold or greater $(P<0.01)$ between $\mathrm{P} 1$ and $\mathrm{P} 14$ cardiac macrophages 3 days following MI. Red indicates upregulated genes whereas green indicates downregulated genes. Functional groups of regulated genes are color coded, and soluble factors are indicated by asterisks.

(Arg1) was the most dramatically upregulated, while 2 of the $3 \mathrm{M} 1$ markers were unchanged or decreased. In contrast, following MI at P1, both types of markers were modestly regulated in the heart, with no clear bias toward M1 or M2.

To gain a more comprehensive understanding of the cardiac monocyte/macrophage genes important for regeneration, we assessed the transcriptional profiles of sorted monocytes/macrophages from hearts of mice 3 days following MI at P1 or P14 by microarray. Using the same staining and gating strategy outlined in Figure 1A, quantitative real-time PCR (qPCR) analysis of RNA isolated from the sorted mononuclear phagocyte population showed enrichment for the expression of monocyte/macrophage-specific genes and depletion in expression of fibroblast genes compared with RNA from the negatively sorted cell population (Supplemental Figure 4A). The analysis of triplicates for each sample allowed us to identify 42 genes that were significantly upregulated in cardiac monocytes/macrophages from mice 3 days following MI at P1 compared with P14 and 41 genes that were downregulated (2-fold or greater cutoff, $P<0.01)$. A majority of the transcripts that were significantly upregulated in P1 samples participate in inflammation or immune function, angiogenesis, or the oxidative stress response (Figure 5B). Furthermore, gene ontology cluster analysis revealed a striking enrichment for genes coding for known or predicted soluble factors (16 of 42). Interestingly, individual M2 genes were significantly higher in both the P1 and P14 groups, suggesting that neither population fits linearly into M1 or M2 polarization. Other genes significantly higher in monocytes/macrophages isolated from hearts following MI at P14 included MHC class II molecules, genes related to immune function, and $\mathrm{G}$ protein-coupled receptors. Of note, only one soluble factor (IL10) was increased in the P14 monocytes/macrophages. The most robustly regulated genes are identified in Supplemental Figure 4B.

Collectively, the gene expression data suggest that while phages. To understand how monocytes/macrophages participate in cardiac regeneration, we sought to identify phenotypic differences between populations from P1 and P14 mice.

Macrophages can be subdivided into M1 or M2 populations based on their expression of multiple markers. We used real-time PCR analysis to compare expression of M1 genes (Cd86, Fcgr1, and Nos2) and M2 genes (Chi3l3 and Arg1) in the heart at 3 days after MI (Figure 5A). Following MI of P14 mice, the M2 marker arginase 1 both P1 and P14 mice mount a monocyte/macrophage response following MI, the magnitude and polarization of the response varies. The differences in M1/M2 marker expression and in the transcriptional profiles of sorted monocyte/macrophage populations likely reflect functional differences. This suggests that monocytes/ macrophages from P1 mice may have regenerative functions unique from the profibrotic and scar-forming activities of adult monocytes/macrophages. 

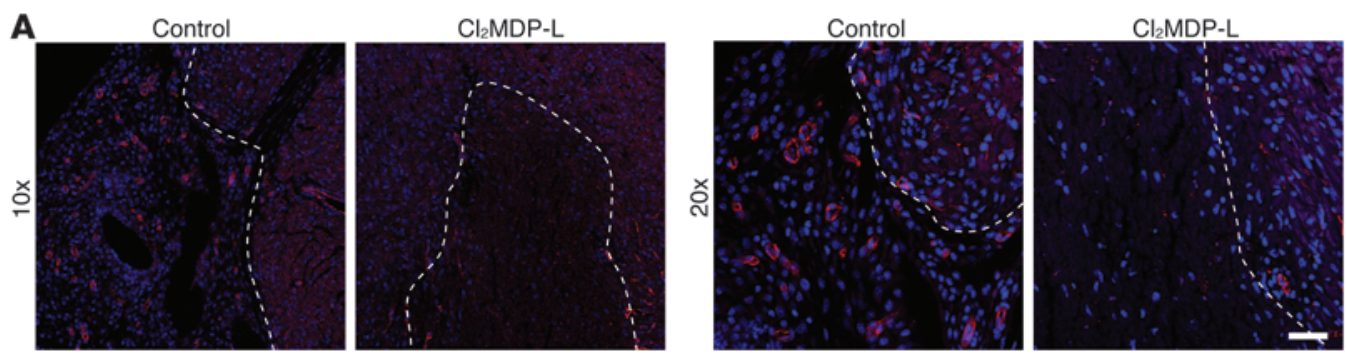

B

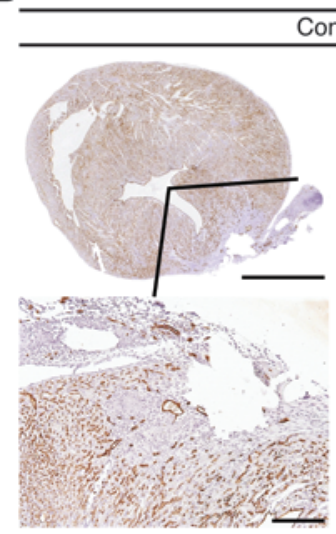

Day 7 after MI

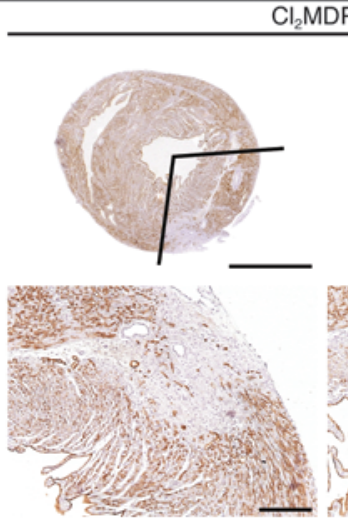

$\mathrm{Cl}_{2}$ MDP-L

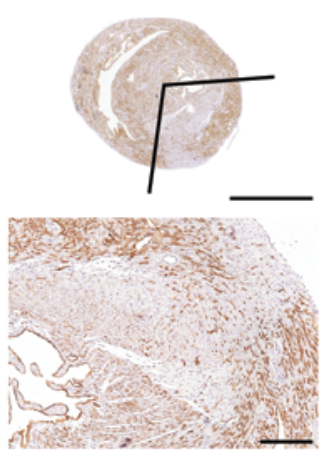

C

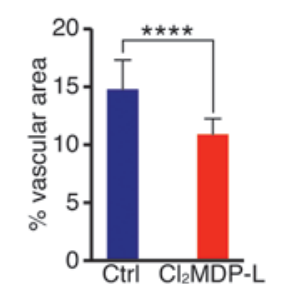

D Day 21 after MI

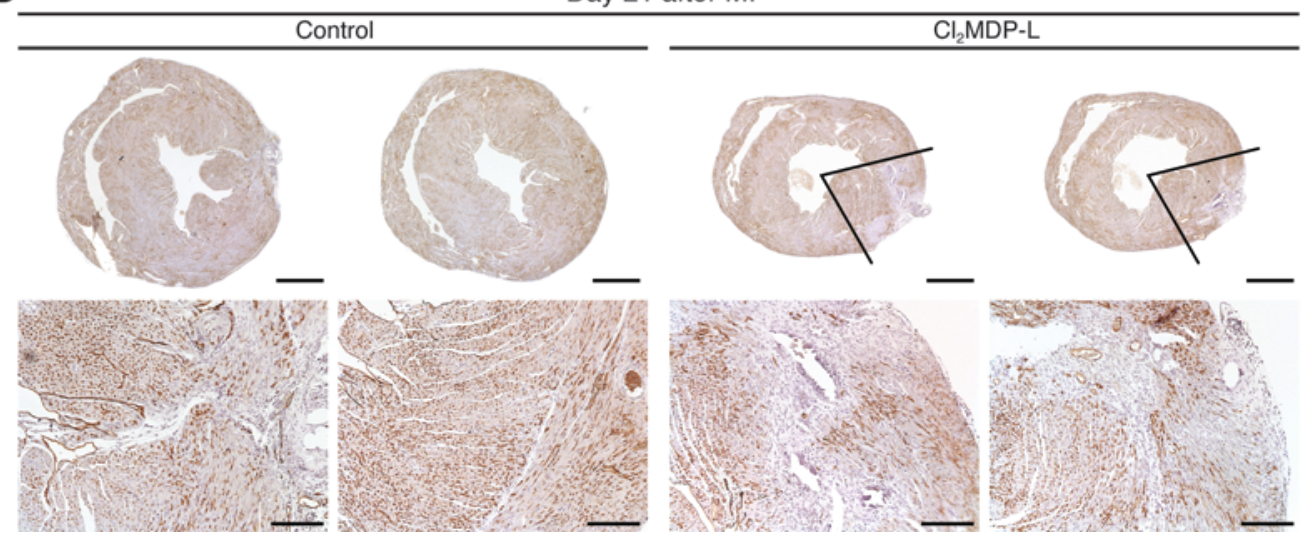

\section{Figure 6}

Angiogenesis is impaired in monocyte/macrophage-depleted neonates following MI. (A) Sections from control or Cl $2 \mathrm{MDP}$-L-treated hearts stained with PECAM (red) and Hoechst (blue) to label endothelial cells and nuclei, respectively, 7 days after MI. Vessels are visible in infarct areas (marked by dashed lines) in controls but not in the $\mathrm{Cl}_{2} \mathrm{MDP}-\mathrm{L}$ group. Original magnification, $\times 10$ (top row); $\times 20$ (bottom row). (B-D) The vasculature was visualized by endomucin immunohistochemistry (brown) on serial heart sections starting below the ligature and progressing toward the apex of control and $\mathrm{Cl}_{2}$ MDP-L-treated neonates at (B) 7 and (D) 21 days after MI. (B) Two serial heart sections for each mouse show newly forming vessels invading the $\mathrm{IZ}$ in control but not $\mathrm{Cl}_{2} \mathrm{MDP}-\mathrm{L}-$ treated neonates, and lines indicate area used to quantify vessel density. (C) Significantly fewer neovessels are present in $\mathrm{Cl}_{2} \mathrm{MDP}$-L-treated mice compared with controls. Data are mean \pm SEM. ${ }^{\star \star \star \star} P<0.0001$. (D) At 21 days after MI, control mice have new myocardia that contain abundant endomucin-positive vasculature, while heart sections from $\mathrm{Cl}_{2} \mathrm{MDP}$-L-treated mice contain areas devoid of new vessels. Lines indicate area lacking vessels. Scale bars: $1 \mathrm{~mm}$ (top rows); $200 \mu \mathrm{m}$ (bottom rows).

Cardiomyocyte proliferation and angiogenesis in macrophage-depleted neonates. Lineage-tracing studies have demonstrated that cardiac regeneration in neonatal mice $(2,3)$ and zebrafish involves proliferation of preexisting cardiomyocytes (37). In order to understand how monocytes/macrophages regulate regeneration, we examined the ability of neonatal cardiomyocytes to proliferate following $\mathrm{MI}$ in the monocyte/macrophage-depleted model. First, heart weights from control and $\mathrm{CL}_{2} \mathrm{MDP}-\mathrm{L}-$ treated mice were compared relative to body weight after sham operation or MI and showed no significant differences (Supplemental Figure $5 \mathrm{~A})$. Serial heart sections from control or $\mathrm{CL}_{2} \mathrm{MDP}-\mathrm{L}-$ treated mice costained for $\mathrm{pH} 3$ and Troponin $\mathrm{T}$ showed that the number of $\mathrm{pH} 3^{+}$cardiomyocytes was not different between macrophage-depleted and control mice at day 7 after MI (Supplemental Figure 5B), suggesting that cardiomyocyte proliferation is not directly controlled by monocytes/macrophages. 


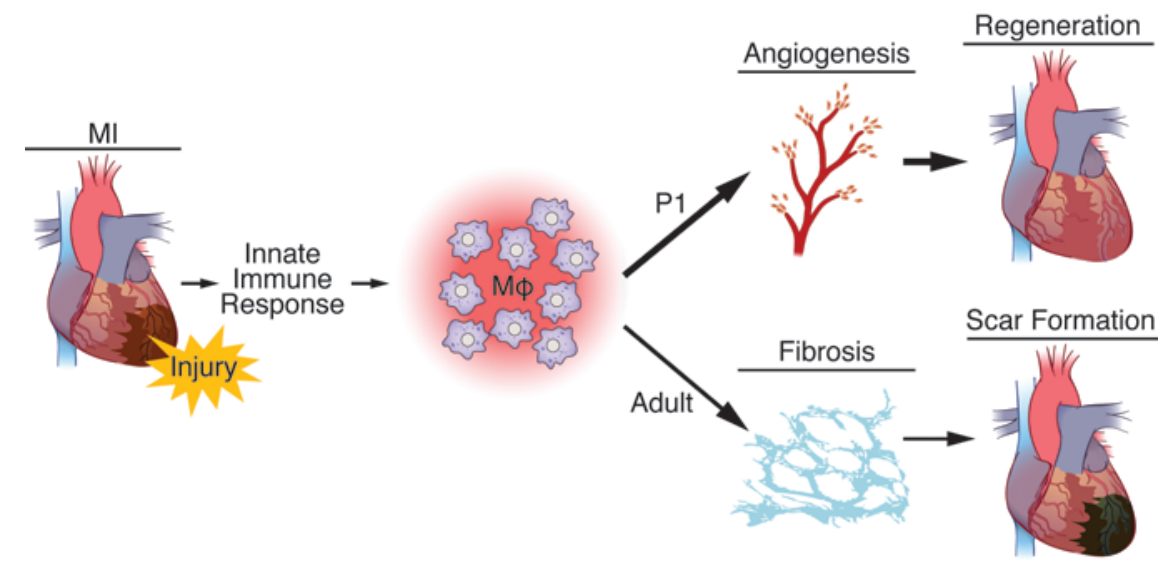

Figure 7

Model demonstrating the dual role of macrophages in regeneration (as in P1 mice) or scar formation (as in adult mice) following MI. Following $\mathrm{MI}$, the innate immune response to cardiac injury involves activation and recruitment of macrophages in the heart, in which they function to clear cellular debris and secrete soluble factors. Macrophage populations promote regenerative processes, such as angiogenesis, in P1 mice, while work done previously in adult mice shows they mediate fibrosis and other aspects of scar formation.

The reestablishment of adequate blood flow to the ischemic and newly forming myocardium is a key aspect of heart regeneration following MI (3). To determine whether monocytes/macrophages mediate new blood vessel formation or angiogenesis, we visualized the vasculature in heart sections from control or $\mathrm{CL}_{2} \mathrm{MDP}-\mathrm{L}-$ treated neonates by PECAM staining (Figure 6A). Confocal images at low and high magnification revealed numerous PECAM-positive vessels in both the border zone and infarct area of control mice 7 days following MI at P1. In monocyte/ macrophage-depleted hearts, PECAM staining was sparse in the IZ (Figure 6A). Neovascularization was also assessed in P1 control or $\mathrm{CL}_{2} \mathrm{MDP}-\mathrm{L}-$ treated hearts at 7 and 21 days after MI by immunohistochemistry and morphometric analysis of endomucin staining (Figure 6, B-D). In control hearts, newly forming vessels were visible in the regenerating infarct by day 7 after MI; serial sections showed vessels invading areas in which new myocardia were actively replacing the infarct and complete vessel coverage in areas distal to the ligation in which the myocardia had already been completely replaced (Figure 6B). Morphometric analysis of the vascularized area showed a significant increase in control hearts compared with that in $\mathrm{CL}_{2} \mathrm{MDP}-\mathrm{L}$-treated hearts 7 days following MI (Figure 6C). By day 21, neovascularization was complete throughout all sections in control mice (Figure 6D). Neonates given $\mathrm{CL}_{2} \mathrm{MDP}-\mathrm{L}$ had some endomucin staining within the infarct, but the lack of additional vessels in areas distal to the ligation at 7 days after MI (Figure 6B) or in all sections below the ligature at 21 days after MI (Figure 6D) suggested that the angiogenic response was incomplete. Together, these data suggest a model in which P1 monocytes/macrophages differ from nonregenerative monocytes/macrophages and directly or indirectly enhance cardiac regeneration via control of angiogenesis (Figure 7).

\section{Discussion}

We present data that implicates the immune system in mammalian cardiac regeneration. We demonstrate that the age-dependent loss of cardiac regenerative potential following MI correlates with differences in the immune response and show that monocytes/ macrophages are essential for heart regeneration. Interestingly, cardiomyocyte proliferation was not dependent on monocytes/ macrophages, suggesting that, while integral to replenishing lost myocardium, it is only one component of the regenerative response. Instead, defects in neovascularization following cardiac injury in monocyte/macrophage-depleted neonates imply that P1 monocytes/macrophages directly or indirectly influence neovascularization during cardiac regeneration.

Monocytes/macrophages in regeneration versus scar formation. Monocytes/macrophages are phagocytic cells central to homeostasis and inflammation via the clearance of apoptotic cells and the production of soluble factors that can shape the immune response and facilitate tissue remodeling. In the proposed model (Figure 7), the regenerative monocyte/macrophage response in neonatal mice $(<\mathrm{P} 7)$ differs from the response in older mice $(\geq \mathrm{P} 7)$ in magnitude, polarization, and transcriptional profile (Figures 1, 2, and 5). Consequently, $\mathrm{P} 1$ monocytes/macrophages promote angiogenesis that is essential for cardiac regeneration, while previous studies show that adult-like monocytes/macrophages produce soluble factors that stimulate fibrosis and establish a scar (29).

In adult tissues, inflammation is central to fibrosis and other processes that heal the injury but impede regeneration. However, effective cardiac repair requires an orchestrated response, and activation of monocytes/macrophages following cardiac injury has both beneficial and deleterious effects on scar formation. Following cryoinjury in adult mice depleted of monocytes/macrophages with clodronate liposomes, lack of collagen deposition, failed clearance of necrotic cells, and reduced angiogenesis impair scar formation, leading to rupture and increased mortality (24). Also, optimal healing from MI requires a precise balance of $\mathrm{Ly}-6 \mathrm{Chi}$ and $\mathrm{Ly}-6 \mathrm{C}^{\text {lo }}$ monocytes to remove debris and form a scar that is compatible with heart function. Conditions that often accompany or precede MI, such as atherosclerosis, obesity, and diabetes, have inflammatory components that disrupt this balance, causing impaired healing (38).

Regeneration supersedes wound healing and scar formation in P1 neonatal hearts. Cardiomyocyte cell cycle withdrawal clearly plays a major role in defining the regenerative window in the neonatal mouse heart. However, our data show that neonatal monocytes/macrophages are also critical for vascularization of 
regenerated neonatal myocardium, a role that differs from their profibrotic function in adulthood. Myeloid cells exert many proangiogenic roles during development and tissue repair, such as production of growth factors like VEGF, physically assisting endothelial tip fusion, or providing temporary scaffolds for vascular expansion (39). It is likely that P1 monocytes/macrophages are unique in their ability to foster neovascularization without simultaneously perpetuating cell damage through protease production or promoting fibrosis.

Macrophage heterogeneity. Tissue macrophages are derived from monocytes, as they enter tissue from the periphery or from local proliferation of resident cells $(40,41)$. Here, we focus on both monocytes and macrophages, given that many of the macrophages identified in the heart and spleen by our analysis may be monocyte derived. Furthermore, while our sorting strategy is standard for identification of monocytes/macrophages, we cannot rule out the potential presence of a small subset of CD $11 b^{+}$ DCs in these populations.

Monocytes/macrophages exhibit considerable heterogeneity that is not yet fully understood. Our expression profiling on sorted cardiac monocytes/macrophages from P1 and P14 mice highlights this diversity and revealed differences between regenerative and nonregenerative monocytes/macrophages that, in addition to the expected inflammatory-related genes, included expression of several soluble factors, angiogenesis genes, and oxidative stress responders. Given that phenotypic differences in monocyte/ macrophage populations often reflect functional variation that influences disease (42), it is likely that P1 cells may perform different functions than their P14 (and adult) counterpoints.

The link between regeneration and immunity - therapeutic implications. A link between development of an "advanced" or mammalian-like immune system and the loss of regenerative capacity has been recognized for decades (30). Studies with antiinflammatory and proinflammatory agents demonstrate that inflammation controls regeneration in Xenopus hind limb (30) and inflammation is necessary and sufficient to induce zebrafish neurogenesis following injury (43). A recent study in axolotls, salamanders that retain regenerative capacity throughout life, showed a requirement for macrophages in limb regeneration following amputation (31). Examples in mammals suggesting that monocytes/macrophages can have regenerative effects are also beginning to emerge. In skeletal muscle, regeneration following toxic injury depends on monocyte/macrophage stimulation of myogenic proliferation (33) and remyelination in the aging central nervous system is stimulated by recruitment of young monocytes (32).

By using a novel model of mammalian heart regeneration, our studies provide direct evidence that the immune system, and specifically monocytes/macrophages, play a role in heart regeneration. Given that cardiac regeneration can only occur within a narrow developmental window in mammals, the features that define this window immunologically will be of particular therapeutic relevance. However, regeneration clearly involves additional processes that also define the regenerative window, such as cardiomyocyte proliferative capacity and cell cycle exit (3). Thus, there is likely interplay between the developmental state of cardiomyocytes and the neonatal immune response.

By identifying differences between the immune response during cardiac regeneration and that during scar formation and studying neonatal monocytes/macrophages, it may be possible to develop therapies to poise adult monocytes/macrophages to enhance car- diac regeneration. While there are positive and negative aspects of monocyte/macrophage function in the wound healing response, their requirement in regeneration is clearly different. Precise calibration of the immunological environment will be necessary to preserve positive monocyte/macrophage effects, such as angiogenesis, while minimizing negative components of the inflammatory response.

\section{Methods}

Neonatal MI model and clodronate liposome treatment. MI surgeries were performed on neonatal mice (ICR/CD-1 strain, Charles River Laboratories), as previously described (3). Briefly, after anesthetizing on ice, lateral thoracotomy was performed and a 6-0 prolene suture (Ethicon) was tied through the left anterior descending coronary artery to induce infarction. Sham-operated mice underwent the same procedure without left anterior descending ligation. After surgery, incisions were sutured with a 6-0 nonabsorbable prolene suture, and neonates were allowed to recover under a heat lamp for several minutes. For MI surgeries at P14, mice were anesthetized with isoflurane, followed by endotracheal intubation for ventilation by using a small animal ventilator (Harvard Apparatus).

Clodronate liposomes were obtained from the nonprofit consortium Foundation Clodronate Liposomes and stored for 4 to 6 weeks at $4^{\circ} \mathrm{C}$ during use.

Briefly, room temperature $\mathrm{CL}_{2} \mathrm{MDP}-\mathrm{L}(10 \mu \mathrm{l} / \mathrm{g})$ was administered i.v. 4 hours before MI and again at 1, 3, and 6 days after MI. Due to limitations in IV access, the final dose was administered by intraperitoneal injection.

RT-PCR and PPCR analysis. Total RNA was isolated from mouse hearts and spleens using TRIzol (Invitrogen), and RT-PCR was performed using random hexamer primers with the SuperScript III Kit (Invitrogen). qPCR was performed using Sybr probes with the following sequences: Arg1for 5'-GGAACCCAGAGAGAGCATGA-3'; rev 5'-TTTTCCAGCAGACCAGCTTT-3'; Cd86-for 5'-CTTACGGAAGCACCCATGAT-3'; rev 5'-CCCATTGAAATAAGCTTGCG-3'; Cd64-for 5'-AGGTTCCTCAATGCCAAGTG-3'; rev 5'-ATTCTTCCATCCGTGACACC-3'; iNOS-for 5'-GAAGAAAACCCCTTGTGCTG-3'; rev 5'-TCCAGGGATTCTGGAACATT-3'; Chi313-for 5'-AGGAGCAGGAATCATTGACG-3'; rev 5'-TTTCTCCAGTGTAGCCATCCTT-3'; Ccl2-for 5'-AGGTCCCTGTCATGCTTCTG-3'; rev 5'-GGGATCATCTTGCTGGTGAA-3'; Ccl3-for 5'-CTGCCCTTGCTGTTCTTCTC-3'; rev 5'-GATGAATTGGCGTGGAATCT-3'; Ccl4-for 5'-AACACCATGAAGCTCTGCGT-3'; rev 5'-GAAACAGCAGGAAGTGGGAG-3'; Cxcl2-for 5'-CAAAAAGTTTGCCTTGACCC-3'; rev 5'-CTTTGGTTCTTCCGTTGAGG-3'; Emr1-for 5'-GGATGTACAGATGGGGGATG-3'; rev 5'-GTCTGTGGTGTCAGTGCAGG-3'; Cd14-for 5'-GGAAGCCAGAGAACACCATC-3'; rev 5'-CCAGAAGCAACAGCAACAAG-3'; and Thy1-for 5'-AACTCTTGGCACCATGAACC-3'; rev 5'-AGTCCAGGCGAAGGTTTTG- $3^{\prime}$.

Flow cytometry. Single cell suspensions of hearts and spleens from P1 or P14 mice were generated immediately before analysis by flow cytometry, as previously described (44). Briefly, pooled hearts were minced and digested in collagenase/DNAse solution in PBS with $20 \mathrm{mM}$ HEPES $(125 \mathrm{U} / \mathrm{ml}$ collagenase type XI, $60 \mathrm{U} / \mathrm{ml}$ hyaluronidase, $60 \mathrm{U} / \mathrm{ml}$ DNase 1 , and $450 \mathrm{U} / \mathrm{ml}$ collagenase type I) for 30 minutes at $37^{\circ} \mathrm{C}$ with rotation. Digested samples were passed through a $70-\mu \mathrm{m}$ filter, washed, and suspended in FACS buffer (PBS 1\% FBS, 0.3\% BSA) for staining. Spleens were disrupted with the blunt end of a 1-ml syringe and passed through a cell strainer. Samples were stained with antibodies from BD Biosciences: CD11c FITC, I-A ${ }^{b}$ FITC, CD4 FITC, Ly-6C PE, CD25 PE, Ly-6G PerCP-Cy5.5, TCR $\beta$ PerCP-Cy5.5, CD11b $\mathrm{APC}$, and B220 APC. F4/80 FITC was from eBioscience. Staining and sample analysis were all performed by standard procedure on a FACSCalibur.

Cell sorting and microarray analysis. Single cell suspensions were obtained from pooled hearts at 3 days following MI at P1 or P14 by collagenase digestion and stained with antibodies to distinguish mononuclear phago- 
cytes (described above). The mononuclear phagocyte population and remaining cells were separated by FACS using a MoFlo (Beckman Coulter) high-speed sorter. Microarray analysis was performed on RNA samples extracted from the sorted populations by the University of Texas Southwestern Microarray Core Facility, which were analyzed for gene expression profiles using the Illumina Mouse-6 Beadchip (Illumina). Briefly, cDNA was column purified following synthesis according to the manufacturer's protocol and then used for in vitro transcription to generate cRNA. After purification, cRNA quality was checked using an Agilent 2100 Bioanalyzer before proceeding with hybridization. Samples were hybridized overnight at $58^{\circ} \mathrm{C}$ with rocking, washed, blocked, and then stained with SA-Cy3. Slides were dried and scanned using the Direct Hybridization program on the BeadArray Reader. A fold-change cutoff of 2.0 was considered significant. Data are available from GEO (accession no. GSE54530).

Transthoracic echocardiography. Cardiac function and heart dimensions were evaluated by 2-dimensional echocardiography using a Visual Sonics Vevo 2100 Ultrasound (Visual Sonics) on conscious mice. M-mode tracings were used to measure anterior and posterior wall thicknesses at end diastole and end systole. Left ventricular internal diameter (LVID) was measured as the largest anteroposterior diameter in either diastole (LVIDd) or systole (LVIDs). A single observer blinded to mouse experimental groups performed echocardiography and data analysis. Fractional shortening (FS) was calculated according to the following formula: FS $(\%)=[($ LVIDd - LVIDs $) /$ LVIDd $] \times 100$.

Histology and immunostaining. Heart and spleen tissues were fixed in $4 \%$ paraformaldehyde, embedded in paraffin, and sectioned at $5-\mu \mathrm{m}$ intervals. H\&E, Masson's trichrome, and Picrosirius red stains were performed using standard procedures. Immunohistochemistry for endomucin to mark vasculature and double immunofluorescence staining for phospho-histone $\mathrm{H} 3$ and cardiac troponin $\mathrm{T}$ was performed as previously described (2). PECAM staining was performed using standard methods and imaged by confocal microscopy.

For immunohistochemical staining, the following antibodies were used: F4/80 (1:200, Serotec); Mac-3 (1:200, BD Pharmingen); and NIMP-R14 (1:200, Abcam). Cryosections were fixed for 10 minutes in ice-cold acetone. Slides were treated with $0.3 \% \mathrm{H}_{2} \mathrm{O}_{2}$ for 20 minutes before blocking in $10 \%$ rabbit serum for 1 hour at room temperature. Slides were incubated with unconjugated primary antibodies listed above overnight at $4^{\circ} \mathrm{C}$.
Slides were washed 3 times in PBS and incubated with rabbit biotinylated anti-rat secondary (1:200, Vector) for 30 minutes at room temperature, followed by SA-HRP (1:500, Vector) for 30 minutes at room temperature. Staining was visualized with the DAB Kit (Dako) and counterstained with hematoxylin before mounting.

Statistics. Results are expressed as the mean \pm SEM. We used a 2-tailed, unpaired Student's $t$ test for all pair-wise comparisons (GraphPad Prism version 5). $P$ values of less than 0.05 were considered significant.

Study approval. All animal procedures were approved by the Institutional Animal Care and Use Committee at University of Texas Southwestern Medical Center.

\section{Acknowledgments}

We are grateful to Chandrashekhar Pasare for scientific discussions and reading of the manuscript, Jimin Pei for assistance with microarray analysis, Lillian Sutherland for technical support, and Jose Cabrera for graphics. We thank John Shelton, James Richardson, and the University of Texas Southwestern Histology Core for assistance with histology. Flow cytometry was performed with assistance from Angela Mobley at the University of Texas Southwestern Flow Cytometry Core Facility. This work was supported by grants from the NIH (HL-077439, HL-111665, HL093039, and U01-HL-100401), AHA-Jon Holden DeHaan Foundation (0970518N), Foundation Leducq Networks of Excellence, Cancer Prevention \& Research Institute of Texas, and the Robert A. Welch Foundation (1-0025) for E.N. Olson.

Received for publication July 17, 2013, and accepted in revised form November 21, 2013.

Address correspondence to: Eric N. Olson, Department of Molecular Biology, 5323 Harry Hines Blvd., Dallas, Texas 75390-9148, USA. Phone: 214.648.1187; Fax: 214.648.1196; E-mail: eric.olson@ utsouthwestern.edu.

Enzo R. Porrello's present address is: School of Biomedical Sciences, The University of Queensland, Brisbane, Australia.
1. Minino AM, Murphy SL, Xu J, Kochanek KD Deaths: final data for 2008. Natl Vital Stat Rep. 2011;59(10):1-126.

2. Porrello ER, et al. Transient regenerative potential of the neonatal mouse heart. Science. 2011; 331(6020):1078-1080.

3. Porrello ER, et al. Regulation of neonatal and adult mammalian heart regeneration by the miR-15 family. Proc Natl Acad Sci U S A. 2013;110(1):187-192.

4. Mahmoud AI, Porrello ER. Turning back the cardiac regenerative clock: lessons from the neonate. Trends Cardiovasc Med. 2012;22(5):128-133.

5. Walsh S, Pontén A, Fleischmann BK, Jovinge S. Cardiomyocyte cell cycle control and growth estimation in vivo - an analysis based on cardiomyocyte nuclei. Cardiovasc Res. 2010;86(3):365-373.

6. Li F, Wang X, Capasso JM, Gerdes AM. Rapid transition of cardiac myocytes from hyperplasia to hypertrophy during postnatal development. J Mol Cell Cardiol. 1996;28(8):1737-1746.

7. Garcia AM, Fadel SA, Cao S, Sarzotti M. T cell immunity in neonates. Immunol Res. 2000;22(2-3):177-190.

8. Foss DL, Donskoy E, Goldschneider I. The importation of hematogenous precursors by the thymus is a gated phenomenon in normal adult mice. J Exp Med. 2001;193(3):365-374.

9. Lu LS, Tung J, Baumgarth N, Herman O, Gleimer $\mathrm{M}$, Herzenberg LA. Identification of a germ-line pro-B cell subset that distinguishes the fetal/neo- natal from the adult B cell development pathway. Proc Natl Acad Sci U S A. 2002;99(5):3007-3012.

10. Kincade PW, Owen JJ, Igarashi H, Kouro T, Yokota T, Rossi MI. Nature or nurture? Steady-state lymphocyte formation in adults does not recapitulate ontogeny. Immunol Rev. 2002;187(1):116-125.

11. Marshall-Clarke S, Reen D, Tasker L, Hassan J. Neonatal immunity: how well has it grown up? Immunol Today. 2000;21(1):35-41.

12. Siegrist CA. Neonatal and early life vaccinology. Vaccine. 2001;19(25-26):3331-3346.

13. Adkins B, Leclerc C, Marshall-Clarke S. Neonatal adaptive immunity comes of age. Nat Rev Immunol. 2004;4(7):553-564.

14. Levy O. Innate immunity of the newborn: basic mechanisms and clinical correlates. Nat Rev Immunol. 2007;7(5):379-390.

15. Firth MA, Shewen PE, Hodgins DC. Passive and active components of neonatal innate immune defenses. Anim Health Res Rev. 2005;6(2):143-158.

16. Aittoniemi J, et al. Age-dependent variation in the serum concentration of mannan-binding protein. Acta Paediatr. 1996;85(8):906-909.

17. Dakic A, et al. Development of the dendritic cell system during mouse ontogeny. J Immunol. 2004;172(2):1018-1027.

18. Coggins M, Rosenzweig A. The fire within: cardiac inflammatory signaling in health and disease. Circ Res. 2012;110(1):116-125
19. Huang GN, et al. C/EBP transcription factors mediate epicardial activation during heart development and injury. Science. 2012;338(6114):1599-1603.

20. Kaikita K, Hayasaki T, Okuma T, Kuziel WA, Ogawa H, Takeya M. Targeted deletion of CC chemokine receptor 2 attenuates left ventricular remodeling after experimental myocardial infarction. Am J Pathol. 2004;165(2):439-447.

21. Hayashidani S, et al. Anti-monocyte chemoattractant protein-1 gene therapy attenuates left ventricular remodeling and failure after experimental myocardial infarction. Circulation. 2003; 108(17):2134-2140.

22. Maekawa Y, et al. Effect of granulocyte-macrophage colony-stimulating factor inducer on left ventricular remodeling after acute myocardial infarction. J Am Coll Cardiol. 2004;44(7):1510-1520.

23. Morimoto $\mathrm{H}$, et al. Cardiac overexpression of monocyte chemoattractant protein- 1 in transgenic mice prevents cardiac dysfunction and remodeling after myocardial infarction. Circ Res. 2006; 99(8):891-899.

24. van Amerongen MJ, Harmsen MC, van Rooijen N, Petersen AH, van Luyn MJ. Macrophage depletion impairs wound healing and increases left ventricular remodeling after myocardial injury in mice. $\mathrm{Am}$ J Pathol. 2007;170(3):818-829.

25 . Leor J, et al. Ex vivo activated human macrophages improve healing, remodeling, and func- 
tion of the infarcted heart. Circulation. 2006; 114(1 suppl):I94-I100

26. Swirski FK, et al. Identification of splenic reservoir monocytes and their deployment to inflammatory sites. Science. 2009;325(5940):612-616.

27. Leuschner F, et al. Angiotensin-converting enzyme inhibition prevents the release of monocytes from their splenic reservoir in mice with myocardial infarction. Circ Res. 2010;107(11):1364-1373.

28. Nahrendorf $M$, et al. The healing myocardium sequentially mobilizes two monocyte subsets with divergent and complementary functions. J Exp Med. 2007;204(12):3037-3047.

29. Nahrendorf M, Pittet MJ, Swirski FK. Monocytes: protagonists of infarct inflammation and repair after myocardial infarction. Circulation. 2010;121(22):2437-2445.

30. King MW, Neff AW, Mescher AL. The developing Xenopus limb as a model for studies on the balance between inflammation and regeneration. Anat Rec. 2012;295(10):1552-1561.

31. Godwin JW, Pinto AR, Rosenthal NA. Macrophages are required for adult salamander limb regeneration. Proc Natl Acad Sci U S A. 2013;110(23):9415-9420.

32. Ruckh JM, et al. Rejuvenation of regeneration in the aging central nervous system. Cell Stem Cell. 2012;10(1):96-103.

33. Arnold L, et al. Inflammatory monocytes recruited after skeletal muscle injury switch into antiinflammatory macrophages to support myogenesis. J Exp Med. 2007;204(5):1057-1069.

34. Haubner BJ, et al. Complete cardiac regeneration in a mouse model of myocardial infarction. Aging. 2012;4(12):966-977.

35. Waller AH, Sanchez-Ross M, Kaluski E, Klapholz M. Osteopontin in cardiovascular disease: a potential therapeutic target. Cardiol Rev. 2010;18(3):125-131.

36. Biernacka A, Frangogiannis NG. Aging and cardiac fibrosis. Aging Dis. 2011;2(2):158-173.

37. Choi WY, Poss KD. Cardiac regeneration. Curr Top Dev Biol. 2012;100:319-344.

38. Swirski FK, Nahrendorf M. Leukocyte behavior in atherosclerosis, myocardial infarction, and heart failure. Science. 2013;339(6116):161-166.
39. Nucera S, Biziato D, De Palma M. The interplay between macrophages and angiogenesis in development, tissue injury and regeneration. Int J Dev Biol. 2011;55(4-5):495-503.

40. Chow A, Brown BD, Merad M. Studying the mononuclear phagocyte system in the molecular age. Nat Rev Immunol. 2011;11(11):788-798.

41. Geissmann F, Manz MG, Jung S, Sieweke MH, Merad M, Ley K. Development of monocytes, macrophages, and dendritic cells. Science. 2010; 327(5966):656-661.

42. Geissmann F, Jung S, Littman DR. Blood monocytes consist of two principal subsets with distinct migratory properties. Immunity. 2003; 19(1):71-82.

43. Kyritsis N, et al. Acute inflammation initiates the regenerative response in the adult zebrafish brain. Science. 2012;338(6112):1353-1356.

44. Leuschner F, et al. Rapid monocyte kinetics in acute myocardial infarction are sustained by extramedullary monocytopoiesis. J Exp Med. 2012;209(1):123-137. 